\title{
Homogenization of Elastic Plate Equation*
}

\author{
Krešimir Burazin ${ }^{a}$, Jelena Jankov ${ }^{a}$ and Marko Vrdoljak ${ }^{b}$ \\ ${ }^{a}$ Department of Mathematics, University of Osijek \\ Trg Ljudevita Gaja 6, Osijek, Croatia \\ ${ }^{b}$ Department of Mathematics, Faculty of Science, University of Zagreb \\ Bijenička c. 30, Zagreb, Croatia \\ E-mail: kburazin@mathos.hr \\ E-mail: jjankov@mathos.hr \\ E-mail(corresp.): marko@math.hr
}

Received July 28, 2017; revised January 17, 2018; accepted January 19, 2018

\begin{abstract}
We are interested in general homogenization theory for fourth-order elliptic equation describing the Kirchhoff model for pure bending of a thin solid symmetric plate under a transverse load. Such theory is well-developed for second-order elliptic problems, while some results for general elliptic equations were established by Zhikov, Kozlov, Oleinik and Ngoan (1979). We push forward an approach of Antonić and Balenović $(1999,2000)$ by proving a number of properties of $\mathrm{H}$-convergence for stationary plate equation.
\end{abstract}

Keywords: Kirchhoff model of elastic plate, homogenization, H-convergence, correctors.

AMS Subject Classification: 35J30; 74K20; 74Q05.

\section{Introduction}

We consider a homogeneous Dirichlet boundary value problem for a general fourth-order partial differential equation

$$
\left\{\begin{array}{l}
\operatorname{div} \operatorname{div}(\mathbf{M} \nabla \nabla u)=f \text { in } \Omega, \\
u \in \mathrm{H}_{0}^{2}(\Omega)
\end{array}\right.
$$

where $\Omega \subseteq \mathbf{R}^{d}$ is an open and bounded set, and $\mathbf{M}$ is a tensor valued function, which can be understood as a linear operator on the space of all symmetric $d \times d$ real matrices, denoted by Sym.

\footnotetext{
* This work has been supported in part by Croatian Science Foundation under the project
} 9780 WeConMApp.

Copyright (c) 2018 The Author(s). Published by VGTU Press

This is an Open Access article distributed under the terms of the Creative Commons Attribution License (http://creativecommons.org/licenses/by/4.0/), which permits unrestricted use, distribution, and reproduction in any medium, provided the original author and source are credited. 
The weak solution $u$ of (1.1) is defined as a function $u \in \mathrm{H}_{0}^{2}(\Omega)$ satisfying

$$
\left(\forall v \in \mathrm{H}_{0}^{2}(\Omega)\right) \quad \int_{\Omega} \mathbf{M} \nabla \nabla u: \nabla \nabla v d \mathbf{x}={ }_{\mathrm{H}^{-2}(\Omega)}\langle f, v\rangle_{\mathrm{H}_{0}^{2}(\Omega)} .
$$

The problem is elliptic, if we assume that $\mathbf{M}$ is bounded (almost everywhere) and coercive. More precisely, we assume that $\mathbf{M}$ belongs to $\mathfrak{M}_{2}(\alpha, \beta ; \Omega):=$ $\left\{\mathbf{M} \in \mathrm{L}^{\infty}(\Omega ; \mathcal{L}(\operatorname{Sym}, \operatorname{Sym})):(\forall \mathbf{S} \in \operatorname{Sym}) \mathbf{M}(\mathbf{x}) \mathbf{S}: \mathbf{S} \geq \alpha \mathbf{S}: \mathbf{S} \& \mathbf{M}(\mathbf{x})^{-1} \mathbf{S}:\right.$ $\mathbf{S} \geq \frac{1}{\beta} \mathbf{S}: \mathbf{S}$, a.e. $\left.\mathbf{x} \in \Omega\right\}$, where $\beta>\alpha>0$ are given, and : stands for the scalar product on the space Sym. The bounds are chosen in this form to ensure their preservation during the homogenization process, as noted in [11] in the case of stationary diffusion equation.

The well-posedness follows by a standard application of the Lax-Milgram lemma. To be precise, differential operator $\operatorname{div} \operatorname{div}(\mathbf{M} \nabla \nabla \cdot): \mathrm{H}_{0}^{2}(\Omega) \longrightarrow \mathrm{H}^{-2}(\Omega)$ is an isomorphism, i. e. a linear and continuous operator with bounded inverse (the bound depending only on $\Omega$ and $\alpha$ ).

In the two-dimensional case, boundary value problem (1.1) describes the Kirchhoff (also known as Kirchhoff-Love) model for pure bending of a thin, solid symmetric plate clamped at the boundary, under a transverse load $f$. This model can be derived by taking a limit in 3 d elasticity equations with a technique similar to $H$-convergence [8], or by means of Gamma convergence [7]. The plate is assumed to be symmetric with respect to its midplane $\Omega$ and a tensor valued function $\mathbf{M}$ describes its elastic properties (depending on the material properties and the thickness of the plate). In this model, additional symmetry is present, making tensor function $\mathbf{M}$ self-adjoint. Such assumption simplifies the theory, since it is equivalent to consider $G$-convergence $[9,10]$ instead of $H$-convergence. However, in this paper we shall present the general theory (in arbitrary space dimension), ignoring this symmetry assumption.

We are interested in the general (non-periodic) homogenization theory for this equation. Such theory is well developed for second-order elliptic problems, such as the stationary diffusion equation or the system of linearized elasticity, for which the notion of $\mathrm{H}$ - (or G-) convergence has been studied and properties, such as compactness, locality, independence of boundary conditions and convergence of energies, have been established (see [1,14] and references therein). In $[5,15]$, a homogenization of general elliptic system of partial differential equations has been considered, and some of the above properties have been shown in such full generality. However, due to this generality, some of the important properties are missing, while proofs end up being rather complicated.

In this paper we push forward the work of Antonić and Balenović [2,3], where, prompted by possible applications in optimal design problems, a more direct approach to the homogenization of stationary plate equation was considered, and compactness of H-convergence was established. Using Tartar's method of oscillating test functions $[13,14]$, we give simpler proofs for the above mentioned properties of H-convergence for stationary plate equation, and additionally prove a number of results, such as the metrizability and the corrector result.

The paper is organized as follows: we finish introductory section by recalling the definition of $\mathrm{H}$-convergence, the compactness by compensation re- 
sult suited for the stationary plate equation, and the compactness result for $\mathrm{H}$-convergence $[2,3]$. In the second section we prove main properties of $\mathrm{H}$ convergence, including locality, independence of boundary conditions, metrizability of H-topology and convergence of energies, and in the third section we give the corrector result.

Definition 1. A sequence of tensor functions $\left(\mathbf{M}^{n}\right)$ in $\mathfrak{M}_{2}(\alpha, \beta ; \Omega)$ H-converges to $\mathbf{M} \in \mathfrak{M}_{2}\left(\alpha^{\prime}, \beta^{\prime} ; \Omega\right)$ if for any $f \in \mathrm{H}^{-2}(\Omega)$ the sequence of solutions $\left(u_{n}\right)$ of problems

$$
\left\{\begin{array}{l}
\operatorname{div} \operatorname{div}\left(\mathbf{M}^{n} \nabla \nabla u_{n}\right)=f \\
u_{n} \in \mathrm{H}_{0}^{2}(\Omega)
\end{array}\right.
$$

converges weakly to a limit $u$ in $\mathrm{H}_{0}^{2}(\Omega)$, while the sequence $\left(\mathbf{M}^{n} \nabla \nabla u_{n}\right)$ converges to $\mathbf{M} \nabla \nabla u$ weakly in the space $\mathrm{L}^{2}(\Omega ; \mathrm{Sym})$.

This implies that $u$ solves the boundary value problem

$$
\left\{\begin{array}{l}
\operatorname{div} \operatorname{div}(\mathbf{M} \nabla \nabla u)=f, \\
u \in \mathrm{H}_{0}^{2}(\Omega) .
\end{array}\right.
$$

Note that sequences $\left(u_{n}\right)$ and $\left(\mathbf{M}^{n} \nabla \nabla u_{n}\right)$ in the above definition are bounded in $\mathrm{H}_{0}^{2}(\Omega)$ and $\mathrm{L}^{2}(\Omega$; Sym), respectively, and thus converge (on a subsequence). Therefore, H-convergence just makes connection between their limits. Since the existence of $\mathbf{H}$-limit $\mathbf{M}$ is doubtful, the following compactness theorem justifies the previous definition. Moreover, it shows that the bounds in definition of $\mathfrak{M}_{2}(\alpha, \beta ; \Omega)$, which could also be written in many equivalent ways, are chosen in such a way that in the previous definition one actually has $\alpha^{\prime}=\alpha$ and $\beta^{\prime}=\beta$.

Theorem 1. Let $\left(\mathbf{M}^{n}\right)$ be a sequence in $\mathfrak{M}_{2}(\alpha, \beta ; \Omega)$. Then there is a subsequence $\left(\mathbf{M}^{n_{k}}\right)$ and a tensor function $\mathbf{M} \in \mathfrak{M}_{2}(\alpha, \beta ; \Omega)$ such that $\left(\mathbf{M}^{n_{k}}\right) H$ converges to $\mathbf{M}$.

The above theorem can be proved by using the following Lemma $[2,3]$. It also has the key role in proving other properties of homogenization for elastic plate equation.

Lemma 1 [Compactness by compensation result]. Let the following convergences be valid:

$$
w^{n} \longrightarrow w^{\infty} \text { in } \mathrm{H}_{\mathrm{loc}}^{2}(\Omega), \quad \mathbf{D}^{\mathrm{n}} \longrightarrow \mathbf{D}^{\infty} \text { in } \mathrm{L}_{\mathrm{loc}}^{2}(\Omega ; \operatorname{Sym}),
$$

with an additional assumption that the sequence $\left(\operatorname{div} \operatorname{div} \mathbf{D}^{n}\right)$ is contained in a precompact (for the strong topology) set of the space $\mathrm{H}_{\text {loc }}^{-2}(\Omega)$. Then we have

$$
\mathbf{E}^{n}: \mathbf{D}^{n} \stackrel{*}{\longrightarrow} \mathbf{E}^{\infty}: \mathbf{D}^{\infty}
$$

in the space of Radon measures, where we denote $\mathbf{E}^{n}:=\nabla \nabla w^{n}$, for $n \in \mathbf{N} \cup$ $\{\infty\}$. 


\section{Properties of $\mathrm{H}$-convergence}

In this section we prove the main properties of the H-convergence, which correspond to the similar properties obtained for the stationary diffusion equation. The proofs are commonly based on Tartar's method of oscillating test functions. The relationship between H-convergence and some other types of convergence is studied in the next theorem.

Theorem 2. Let $\left(\mathbf{M}^{n}\right)$ be a sequence of tensors in $\mathfrak{M}_{2}(\alpha, \beta ; \Omega)$ that either converges strongly to a limit tensor $\mathbf{M}^{*}$ in $\mathrm{L}^{1}(\Omega ; \mathcal{L}(\mathrm{Sym}, \mathrm{Sym}))$, or converges to $\mathbf{M}^{*}$ almost everywhere in $\Omega$. Then, $\mathbf{M}^{n}$ also $H$-converges to $\mathbf{M}^{*}$.

Proof. The sequence $\left(\mathbf{M}^{n}\right)$ belongs to $\mathfrak{M}_{2}(\alpha, \beta ; \Omega)$ and therefore it is bounded in $\mathrm{L}^{\infty}(\Omega ; \mathcal{L}($ Sym, Sym $))$. By the Lebesgue dominated convergence theorem $\left(\mathbf{M}^{n}\right)$ converges strongly to $\mathbf{M}^{*}$ in $\mathrm{L}^{p}(\Omega ; \mathcal{L}(\mathrm{Sym}$, Sym $))$, for any $1 \leq p<\infty$. If $u_{n}$ is the solution of

$$
\left\{\begin{array}{l}
\operatorname{div} \operatorname{div}\left(\mathbf{M}^{n} \nabla \nabla u_{n}\right)=f, \\
u_{n} \in \mathrm{H}_{0}^{2}(\Omega),
\end{array}\right.
$$

then the sequence $\left(u_{n}\right)$ is bounded in $\mathrm{H}_{0}^{2}(\Omega)$, and therefore (up to a subsequence) it converges weakly to $u \in \mathrm{H}_{0}^{2}(\Omega)$.

Since $\left(\mathbf{M}^{n}\right)$ converges strongly to $\mathbf{M}^{*}$ in $\mathrm{L}^{2}(\Omega ; \mathcal{L}(\mathrm{Sym}, \mathrm{Sym}))$ and $\left(\nabla \nabla u_{n}\right)$ converges to $\nabla \nabla u$ weakly in $\mathrm{L}^{2}(\Omega ;$ Sym $)$, we conclude that $\boldsymbol{\sigma}_{n}:=\mathbf{M}^{n} \nabla \nabla u_{n}$ converges weakly to $\boldsymbol{\sigma}=\mathbf{M}^{*} \nabla \nabla u$ in $\mathrm{L}^{1}(\Omega$; Sym $)$, and thus also in $\mathrm{L}^{2}(\Omega$; Sym), as sequence $\left(\boldsymbol{\sigma}_{n}\right)$ is bounded in this space.

The homogenized equation has a unique solution in $\mathrm{H}_{0}^{2}(\Omega)$, so every subsequence of $\left(u_{n}\right)$ converges to the same limit $u$ and this implies that the entire sequence $\left(u_{n}\right)$ converges to $u$. Since $f \in \mathrm{H}^{-2}(\Omega)$ is arbitrary, it follows that $\left(\mathbf{M}^{n}\right)$ H-converges to $\mathbf{M}^{*}$.

Theorem 3 [Irrelevance of boundary conditions]. Let $\left(\mathbf{M}^{n}\right)$ be a sequence of tensors in $\mathfrak{M}_{2}(\alpha, \beta ; \Omega)$ that $H$-converges to $\mathbf{M}^{*}$. For any sequence $\left(z_{n}\right)$ such that

$$
\operatorname{div} \operatorname{div}\left(\mathbf{M}^{n} \nabla \nabla z_{n}\right)=\begin{array}{rll}
z_{n} & \longrightarrow z & \text { in } \mathrm{H}_{\mathrm{loc}}^{2}(\Omega), \\
f_{n} & \longrightarrow f & \text { in } \mathrm{H}_{\mathrm{loc}}^{-2}(\Omega),
\end{array}
$$

the weak convergence $\mathbf{M}^{n} \nabla \nabla z_{n} \rightarrow \mathbf{M}^{*} \nabla \nabla z$ in $\mathrm{L}_{\text {loc }}^{2}(\Omega ;$ Sym $)$ holds.

Proof. Let $\omega$ be an open set compactly embedded in $\Omega$. The sequence $\left(z_{n}\right)$ is bounded in $\mathrm{H}^{2}(\omega)$, implying that $\left(\mathbf{M}^{n} \nabla \nabla z_{n}\right)$ is bounded in $\mathrm{L}^{2}(\omega ;$ Sym). If we denote $\boldsymbol{\sigma}_{n}:=\mathbf{M}^{n} \nabla \nabla z_{n}$, we can pass to a weakly convergent subsequence such that $\boldsymbol{\sigma}_{n} \rightarrow \boldsymbol{\sigma}$ in $\mathrm{L}^{2}(\omega ;$ Sym $)$. Since $\omega \Subset \Omega$, there exists $\varphi \in \mathrm{C}_{c}^{\infty}(\Omega)$ such that $\left.\varphi\right|_{\omega}=1$. For arbitrary $\mathbf{N} \in$ Sym, we define

$$
w(\mathbf{x}):=\frac{1}{2} \varphi(\mathbf{x}) \mathbf{N} \mathbf{x} \cdot \mathbf{x}, \quad g:=\operatorname{div} \operatorname{div}(\mathbf{M} \nabla \nabla w) \in \mathrm{H}^{-2}(\Omega) .
$$

Let $\left(w_{n}\right)$ be a sequence of solutions to

$$
\left\{\begin{array}{l}
\operatorname{div} \operatorname{div}\left(\mathbf{M}^{n} \nabla \nabla w_{n}\right)=g, \\
w_{n} \in \mathrm{H}_{0}^{2}(\Omega) .
\end{array}\right.
$$


Since $\left(\mathbf{M}^{n}\right)$ H-converges to $\mathbf{M}^{*}$, the following holds:

$$
w_{n} \longrightarrow w \text { in } \mathrm{H}_{0}^{2}(\Omega), \quad \mathbf{M}^{n} \nabla \nabla w_{n} \longrightarrow \mathbf{M}^{*} \nabla \nabla w \text { in } \mathrm{L}^{2}(\Omega ; \operatorname{Sym}) .
$$

By coercivity of $\mathbf{M}^{n}$ we have

$$
\left(\mathbf{M}^{n} \nabla \nabla z_{n}-\mathbf{M}^{n} \nabla \nabla w_{n}\right):\left(\nabla \nabla z_{n}-\nabla \nabla w_{n}\right) \geq 0 \quad \text { a. e. in } \Omega,
$$

which, after passing to the limit and using the compactness by compensation result, becomes

$$
\left(\boldsymbol{\sigma}-\mathbf{M}^{*} \nabla \nabla w\right):(\nabla \nabla z-\nabla \nabla w) \geq 0 \quad \text { a. e. in } \Omega .
$$

If we consider the previous inequality only in $\omega$, we have:

$$
\left(\boldsymbol{\sigma}-\mathbf{M}^{*} \mathbf{N}\right):(\nabla \nabla z-\mathbf{N}) \geq 0 \quad \text { a. e. in } \omega .
$$

For any joint Lebesgue point $\mathbf{x}_{0} \in \omega$ of $\nabla \nabla z, \boldsymbol{\sigma}$ and $\mathbf{M}^{*}$, let $\mathbf{N}=\nabla \nabla z\left(\mathbf{x}_{0}\right)+t \mathbf{O}$, where $\mathbf{O} \in$ Sym and $t \in \mathbf{R}^{+}$are arbitrary. Now (2.1) yields

$$
\left(\boldsymbol{\sigma}\left(\mathbf{x}_{0}\right)-\mathbf{M}^{*}\left(\mathbf{x}_{0}\right) \nabla \nabla z\left(\mathbf{x}_{0}\right)-t \mathbf{M}^{*}\left(\mathbf{x}_{0}\right) \mathbf{O}\right):(-t \mathbf{O}) \geq 0,
$$

and after dividing this inequality by $-t$ and taking the limit $t \rightarrow 0^{+}$, it follows

$$
\left(\boldsymbol{\sigma}\left(\mathbf{x}_{0}\right)-\mathbf{M}^{*}\left(\mathbf{x}_{0}\right) \nabla \nabla z\left(\mathbf{x}_{0}\right)\right): \mathbf{O} \leq 0 .
$$

By arbitrariness of $\mathbf{O} \in$ Sym, the equality $\boldsymbol{\sigma}\left(\mathbf{x}_{0}\right)=\mathbf{M}^{*}\left(\mathbf{x}_{0}\right) \nabla \nabla z\left(\mathbf{x}_{0}\right)$ now easily follows, which concludes the proof.

The above theorem implies that the notion of $\mathrm{H}$-convergence is not tied to the prescribed boundary conditions: instead of homogeneous Dirichlet boundary conditions in Definition 1 we can take any boundary conditions which enable well posedness of the boundary value problem. H-convergence also implies the convergence of energies, as stated in the sequel.

Theorem 4 [Energy convergence]. Let $\left(\mathbf{M}^{n}\right)$ be a sequence of tensors in $\mathfrak{M}_{2}(\alpha, \beta ; \Omega)$ that $H$-converges to $\mathbf{M}^{*}$. For any $f \in \mathrm{H}^{-2}(\Omega)$, the sequence $\left(u_{n}\right)$ of solutions to

$$
\left\{\begin{array}{l}
\operatorname{div} \operatorname{div}\left(\mathbf{M}^{n} \nabla \nabla u_{n}\right)=f, \\
u_{n} \in \mathrm{H}_{0}^{2}(\Omega)
\end{array}\right.
$$

satisfies

$$
\begin{aligned}
& \mathbf{M}^{n} \nabla \nabla u_{n}: \nabla \nabla u_{n} \stackrel{*}{\longrightarrow} \mathbf{M}^{*} \nabla \nabla u: \nabla \nabla u \text { in } \mathcal{M}(\Omega), \\
& \int_{\Omega} \mathbf{M}^{n} \nabla \nabla u_{n}: \nabla \nabla u_{n} d \mathbf{x} \longrightarrow \int_{\Omega} \mathbf{M}^{*} \nabla \nabla u: \nabla \nabla u d \mathbf{x},
\end{aligned}
$$

where $u$ is the solution of the homogenized equation

$$
\left\{\begin{array}{l}
\operatorname{div} \operatorname{div}\left(\mathbf{M}^{*} \nabla \nabla u\right)=f, \\
u \in \mathrm{H}_{0}^{2}(\Omega) .
\end{array}\right.
$$


Proof. If we apply the compactness by compensation result, it can easily be seen that $\mathbf{M}^{n} \nabla \nabla u_{n}: \nabla \nabla u_{n} \stackrel{*}{\longrightarrow} \mathbf{M}^{*} \nabla \nabla u: \nabla \nabla u$ in the space of Radon measures, which proves the first statement.

From the weak formulation of given homogeneous Dirichlet boundary value problems we get

$$
\begin{aligned}
\int_{\Omega} \mathbf{M}^{n} \nabla \nabla u_{n}: \nabla \nabla u_{n} d \mathbf{x} & ={ }_{\mathrm{H}^{-2}(\Omega)}\left\langle f, u_{n}\right\rangle_{\mathrm{H}_{0}^{2}(\Omega)}, \\
\int_{\Omega} \mathbf{M}^{*} \nabla \nabla u: \nabla \nabla u d \mathbf{x} & ={ }_{\mathrm{H}^{-2}(\Omega)}\langle f, u\rangle_{\mathrm{H}_{0}^{2}(\Omega)}
\end{aligned}
$$

and since $\left(u_{n}\right)$ converges weakly to $u$ in $\mathrm{H}_{0}^{2}(\Omega)$, we have

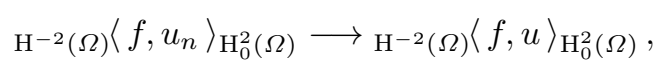

which concludes the proof.

Theorem 5 [Locality of $\mathbf{H}$-convergence]. Let $\left(\mathbf{M}^{n}\right)$ and $\left(\mathbf{O}^{n}\right)$ be two sequences of tensors in $\mathfrak{M}_{2}(\alpha, \beta ; \Omega)$ which $H$-converge to $\mathbf{M}^{*}$ and $\mathbf{O}^{*}$ respectively. Let $\omega$ be an open subset compactly embedded in $\Omega$. If $\mathbf{M}^{n}(\mathbf{x})=\mathbf{O}^{n}(\mathbf{x})$ in $\omega$, then $\mathbf{M}^{*}(\mathbf{x})=\mathbf{O}^{*}(\mathbf{x})$ in $\omega$.

Proof. The proof goes along the same lines as the proof of Theorem 3: since $\omega$ is compactly embedded in $\Omega$, there exists $\varphi \in \mathrm{C}_{c}^{\infty}(\Omega)$ such that $\left.\varphi\right|_{\omega}=1$. For arbitrary $\mathbf{N} \in$ Sym, let us define

$$
w(\mathbf{x}):=\frac{1}{2} \varphi(\mathbf{x}) \mathbf{N} \mathbf{x} \cdot \mathbf{x}, \quad g:=\operatorname{div} \operatorname{div}\left(\mathbf{M}^{*} \nabla \nabla w\right) \in \mathrm{H}^{-2}(\Omega),
$$

and let $w_{n}$ be a sequence of solutions to

$$
\left\{\begin{array}{l}
\operatorname{div} \operatorname{div}\left(\mathbf{M}^{n} \nabla \nabla w_{n}\right)=g, \\
w_{n} \in \mathrm{H}_{0}^{2}(\Omega) .
\end{array}\right.
$$

Since $\left(\mathbf{M}^{n}\right)$ H-converges to $\mathbf{M}^{*}$, it follows that

$$
w_{n} \longrightarrow w \text { in } \mathrm{H}_{0}^{2}(\Omega), \quad \mathbf{M}^{n} \nabla \nabla w_{n} \longrightarrow \mathbf{M}^{*} \nabla \nabla w \text { in } \mathrm{L}^{2}(\Omega ; \mathrm{Sym}) .
$$

For sequence $\left(\mathbf{O}^{n}\right)$ we can proceed similarly: for any $\mathbf{S} \in$ Sym we introduce

$$
v(\mathbf{x}):=\frac{1}{2} \varphi(\mathbf{x}) \mathbf{S x} \cdot \mathbf{x}, \quad f:=\operatorname{div} \operatorname{div}\left(\mathbf{O}^{*} \nabla \nabla v\right) \in \mathrm{H}^{-2}(\Omega),
$$

and let $\left(v_{n}\right)$ be a sequence of solutions to

$$
\left\{\begin{array}{l}
\operatorname{div} \operatorname{div}\left(\mathbf{O}^{n} \nabla \nabla v_{n}\right)=f \\
v_{n} \in \mathrm{H}_{0}^{2}(\Omega)
\end{array}\right.
$$

thus obtaining

$$
v_{n} \longrightarrow v \text { in } \mathrm{H}_{0}^{2}(\Omega), \quad \mathbf{O}^{n} \nabla \nabla v_{n} \longrightarrow \mathbf{O}^{*} \nabla \nabla v \text { in } \mathrm{L}^{2}(\Omega ; \mathrm{Sym}) .
$$


By applying the compactness by compensation result, we get

$$
\begin{aligned}
\left(\mathbf{M}^{n} \nabla \nabla w_{n}-\mathbf{O}^{n} \nabla \nabla v_{n}\right):\left(\nabla \nabla w_{n}-\nabla \nabla v_{n}\right) \\
-{ }^{*}\left(\mathbf{M}^{*} \nabla \nabla w-\mathbf{O}^{*} \nabla \nabla v\right):(\nabla \nabla w-\nabla \nabla v)
\end{aligned}
$$

in the space of Radon measures. On $\omega$ we have $\nabla \nabla v=\mathbf{S}$ and $\nabla \nabla w=\mathbf{N}$, so by assumption $\mathbf{O}^{n}=\mathbf{M}^{n}$ in $\omega$, the sequence in (2.2) equals

$$
\mathbf{O}^{n}\left(\nabla \nabla w_{n}-\nabla \nabla v_{n}\right):\left(\nabla \nabla w_{n}-\nabla \nabla v_{n}\right),
$$

which is nonnegative because of the coercivity of $\mathbf{O}^{n}$. Therefore, the limit in (2.2) is also nonnegative, i. e. $\left(\mathbf{M}^{*} \mathbf{N}-\mathbf{O}^{*} \mathbf{S}\right):(\mathbf{N}-\mathbf{S}) \geq 0$ a. e. in $\omega$. If we choose $\mathbf{S}=\mathbf{N}+t \mathbf{Z}, t \in \mathbf{R}^{+}, \mathbf{Z} \in$ Sym, we obtain

$$
\left(\mathbf{M}^{*} \mathbf{N}-\mathbf{O}^{*} \mathbf{N}-t \mathbf{O}^{*} \mathbf{Z}\right):(-t \mathbf{Z}) \geq 0 \quad \text { in } \omega
$$

and after dividing this inequality by $-t$ and letting $t \rightarrow 0^{+}$, we achieve $\left(\mathbf{M}^{*}-\right.$ $\left.\mathbf{O}^{*}\right) \mathbf{N}: \mathbf{Z} \leq 0$. Since $\mathbf{Z}$ and $\mathbf{N}$ are arbitrary, this implies $\mathbf{M}^{*}=\mathbf{O}^{*}$ a. e. in $\omega$.

We can rephrase the previous theorem by stating that values of the homogenized tensor $\mathbf{M}^{*}$ in a region $\omega$ do not depend on values of the sequence $\left(\mathbf{M}^{n}\right)$ outside of this region.

Theorem 6 [Ordering property]. Let $\left(\mathbf{M}^{n}\right)$ and $\left(\mathbf{O}^{n}\right)$ be two sequences of tensors in $\mathfrak{M}_{2}(\alpha, \beta ; \Omega)$ that $H$-converge to the homogenized tensors $\mathbf{M}^{*}$ and $\mathbf{O}^{*}$, respectively. Assume that $\mathbf{M}^{n}$ and $\mathbf{O}^{n}$ are ordered, for each $n \in \mathbf{N}$ :

$$
\mathbf{M}^{n} \boldsymbol{\xi}: \boldsymbol{\xi} \leq \mathbf{O}^{n} \boldsymbol{\xi}: \boldsymbol{\xi}, \quad \boldsymbol{\xi} \in \text { Sym. }
$$

Then the homogenized coefficients are also ordered:

$$
\mathbf{M}^{*} \boldsymbol{\xi}: \boldsymbol{\xi} \leq \mathbf{O}^{*} \boldsymbol{\xi}: \boldsymbol{\xi}, \quad \boldsymbol{\xi} \in \mathrm{Sym} .
$$

Proof. Let us define a sequence $\left(v_{n}\right)$ of oscillating test functions satisfying

$$
v_{n} \longrightarrow \frac{1}{2} \mathbf{N} \mathbf{x} \cdot \mathbf{x} \text { in } \mathrm{H}^{2}(\Omega), \quad \operatorname{div} \operatorname{div}\left(\mathbf{O}^{n} \nabla \nabla v_{n}\right) \longrightarrow g^{\mathbf{O}} \text { in } \mathrm{H}_{\mathrm{loc}}^{-2}(\Omega),
$$

where $\mathbf{N} \in$ Sym is arbitrary. Existence of such a sequence can be established similarly as in the case of stationary diffusion equation $[1,14]$. Note that $\nabla \nabla v_{n} \longrightarrow \mathbf{N}$, and additionally we have $\mathbf{O}^{n} \nabla \nabla v_{n} \longrightarrow \mathbf{0} * \mathbf{N}$ in $\mathrm{L}_{\text {loc }}^{2}(\Omega ;$ Sym $)$, by Theorem 3 .

Similarly, let us take a sequence $\left(w_{n}\right)$ of oscillating test functions satisfying

$$
\begin{aligned}
& w_{n} \longrightarrow \frac{1}{2} \mathbf{N} \mathbf{x} \cdot \mathbf{x} \text { in } \mathrm{H}^{2}(\Omega), \quad \operatorname{div} \operatorname{div}\left(\mathbf{M}^{n} \nabla \nabla w_{n}\right) \longrightarrow g^{\mathbf{M}} \text { in } \mathrm{H}_{\mathrm{loc}}^{-2}(\Omega), \\
& \mathbf{M}^{n} \nabla \nabla w_{n} \longrightarrow \mathbf{M}^{*} \mathbf{N} \text { in } \mathrm{L}_{\mathrm{loc}}^{2}(\Omega ; \mathrm{Sym}) .
\end{aligned}
$$

Since $\mathbf{M}^{n}$ is coercive, we have

$$
\begin{aligned}
& \mathbf{M}^{n} \nabla \nabla w_{n}: \nabla \nabla w_{n}-\mathbf{M}^{n} \nabla \nabla w_{n}: \nabla \nabla v_{n}-\mathbf{M}^{n} \nabla \nabla v_{n}: \nabla \nabla w_{n} \\
& \quad+\mathbf{M}^{n} \nabla \nabla v_{n}: \nabla \nabla v_{n}=\mathbf{M}^{n}\left(\nabla \nabla w_{n}-\nabla \nabla v_{n}\right):\left(\nabla \nabla w_{n}-\nabla \nabla v_{n}\right) \geq 0
\end{aligned}
$$


in $\Omega$. Since $\mathbf{M}^{n} \leq \mathbf{O}^{n}$, it follows that

$$
\begin{aligned}
& \mathbf{M}^{n} \nabla \nabla w_{n}: \nabla \nabla w_{n}-\mathbf{M}^{n} \nabla \nabla w_{n}: \nabla \nabla v_{n} \\
& \quad-\mathbf{M}^{n} \nabla \nabla v_{n}: \nabla \nabla w_{n}+\mathbf{O}^{n} \nabla \nabla v_{n}: \nabla \nabla v_{n} \geq 0 \quad \text { in } \quad \Omega .
\end{aligned}
$$

By applying the compactness by compensation result, we can pass to the limit in each term of the above expression and get

$$
\mathbf{M}^{*} \mathbf{N}: \mathbf{N}-\mathbf{M}^{*} \mathbf{N}: \mathbf{N}-\mathbf{M}^{*} \mathbf{N}: \mathbf{N}+\mathbf{O}^{*} \mathbf{N}: \mathbf{N}=\left(\mathbf{O}^{*}-\mathbf{M}^{*}\right) \mathbf{N}: \mathbf{N} \geq 0 .
$$

Since $\mathbf{N}$ is arbitrary, it follows that $\mathbf{O}^{*} \geq \mathbf{M}^{*}$.

In the following theorem we introduce bounds on homogenized tensor, in the sense of standard order on symmetric tensors. The bounds are given in terms of weak-* limits, representing harmonic and arithmetic mean of the corresponding sequence.

Theorem 7. Let $\left(\mathbf{M}^{n}\right)$ be a sequence of symmetric tensors in $\mathfrak{M}_{2}(\alpha, \beta ; \Omega)$ that H-converges to $\mathbf{M}^{*}$. Assume that

$$
\begin{aligned}
& \mathbf{M}^{n} \stackrel{*}{\longrightarrow} \overline{\mathbf{M}} \quad \text { in } \quad \mathrm{L}^{\infty}(\Omega ; \mathcal{L}(\operatorname{Sym} ; \operatorname{Sym})), \\
& \left(\mathbf{M}^{n}\right)^{-1} \stackrel{*}{\longrightarrow} \underline{\mathbf{M}}^{-1} \text { in } \quad \mathrm{L}^{\infty}(\Omega ; \mathcal{L}(\operatorname{Sym} ; \operatorname{Sym})) .
\end{aligned}
$$

Then the homogenized tensor satisfies

$$
\underline{\mathbf{M}} \boldsymbol{\xi}: \boldsymbol{\xi} \leq \mathbf{M}^{*} \boldsymbol{\xi}: \boldsymbol{\xi} \leq \overline{\mathbf{M}} \boldsymbol{\xi}: \boldsymbol{\xi}, \quad \boldsymbol{\xi} \in \mathrm{Sym}
$$

Proof. As before, let us take a sequence $\left(w_{n}\right)$ of oscillating test functions satisfying

$$
\begin{aligned}
& w_{n} \longrightarrow \frac{1}{2} \mathbf{N} \mathbf{x} \cdot \mathbf{x} \text { in } \mathrm{H}^{2}(\Omega), \quad \operatorname{div} \operatorname{div}\left(\mathbf{M}^{n} \nabla \nabla w_{n}\right) \longrightarrow g^{\mathbf{M}} \text { in } \mathrm{H}_{\mathrm{loc}}^{-2}(\Omega), \\
& \mathbf{M}^{n} \nabla \nabla w_{n} \longrightarrow \mathbf{M}^{*} \mathbf{N} \text { in } \mathrm{L}_{\mathrm{loc}}^{2}(\Omega ; \operatorname{Sym}),
\end{aligned}
$$

where $\mathbf{N} \in$ Sym is an arbitrary matrix. Since $\mathbf{M}^{n}$ is coercive it follows

$$
\mathbf{M}^{n}\left(\nabla \nabla w_{n}-\mathbf{N}\right):\left(\nabla \nabla w_{n}-\mathbf{N}\right) \geq 0
$$

which, by symmetry of $\mathbf{M}^{n}$, is equivalent to

$$
\mathbf{M}^{n} \nabla \nabla w_{n}: \nabla \nabla w_{n}-2 \mathbf{M}^{n} \nabla \nabla w_{n}: \mathbf{N}+\mathbf{M}^{n} \mathbf{N}: \mathbf{N} \geq 0 .
$$

By the compactness by compensation result, passing to the limit gives

$$
\mathbf{M}^{*} \mathbf{N}: \mathbf{N}-2 \mathbf{M}^{*} \mathbf{N}: \mathbf{N}+\overline{\mathbf{M}} \mathbf{N}: \mathbf{N} \geq 0
$$

thus proving inequality $\overline{\mathbf{M}} \geq \mathbf{M}^{*}$, by arbitrariness of $\mathbf{N}$.

Similarly, for $\boldsymbol{\sigma} \in$ Sym, the coercivity of $\left(\mathbf{M}^{n}\right)^{-1}$ implies

$$
\left(\mathbf{M}^{n}\right)^{-1}\left(\mathbf{M}^{n} \nabla \nabla w_{n}-\boldsymbol{\sigma}\right):\left(\mathbf{M}^{n} \nabla \nabla w_{n}-\boldsymbol{\sigma}\right) \geq 0,
$$


which is equivalent to

$$
\mathbf{M}^{n} \nabla \nabla w_{n}: \nabla \nabla w_{n}-2 \nabla \nabla w_{n}: \boldsymbol{\sigma}+\left(\mathbf{M}^{n}\right)^{-1} \boldsymbol{\sigma}: \boldsymbol{\sigma} \geq 0 .
$$

Passing to the limit as before gives

$$
\mathbf{M}^{*} \mathbf{N}: \mathbf{N}-2 \mathbf{N}: \boldsymbol{\sigma}+\underline{\mathbf{M}}^{-1} \boldsymbol{\sigma}: \boldsymbol{\sigma} \geq 0
$$

which for $\boldsymbol{\sigma}=\underline{\mathrm{MN}}$ becomes

$$
\mathbf{M}^{*} \mathbf{N}: \mathbf{N}-2 \underline{\mathbf{M N}}: \mathbf{N}+\underline{\mathbf{M N}}: \mathbf{N} \geq 0,
$$

i.e.

$$
\left(\mathbf{M}^{*}-\underline{\mathbf{M}}\right) \mathbf{N}: \mathbf{N} \geq 0 .
$$

This proves the second inequality, and concludes the proof.

A useful result is that $\mathrm{H}$-convergence defines a metrizable topology on the set $\mathfrak{M}_{2}(\alpha, \beta ; \Omega)$.

Theorem 8. Let $F=\left\{f_{n}: n \in \mathbf{N}\right\}$ be a dense countable family in $\mathrm{H}^{-2}(\Omega), \mathbf{M}$ and $\mathbf{O}$ tensors in $\mathfrak{M}_{2}(\alpha, \beta ; \Omega)$, and $\left(u_{n}\right),\left(v_{n}\right)$ sequences of solutions to

$$
\left\{\begin{array} { l } 
{ \operatorname { d i v } \operatorname { d i v } ( \mathbf { M } \nabla \nabla u _ { n } ) = f _ { n } , } \\
{ u _ { n } \in \mathrm { H } _ { 0 } ^ { 2 } ( \Omega ) }
\end{array} \quad \text { and } \quad \left\{\begin{array}{l}
\operatorname{div} \operatorname{div}\left(\mathbf{O} \nabla \nabla v_{n}\right)=f_{n}, \\
v_{n} \in \mathrm{H}_{0}^{2}(\Omega),
\end{array}\right.\right.
$$

respectively. Then,

$$
d(\mathbf{M}, \mathbf{O}):=\sum_{n=1}^{\infty} 2^{-n} \frac{\left\|u_{n}-v_{n}\right\|_{\mathrm{L}^{2}(\Omega)}+\left\|\mathbf{M} \nabla \nabla u_{n}-\mathbf{O} \nabla \nabla v_{n}\right\|_{\mathrm{H}^{-1}(\Omega ; \mathrm{Sym})}}{\left\|f_{n}\right\|_{\mathrm{H}^{-2}(\Omega)}}
$$

is a metric function on $\mathfrak{M}_{2}(\alpha, \beta ; \Omega)$ and $H$-convergence is equivalent to the convergence with respect to $d$.

Proof. Since $\mathfrak{M}_{2}(\alpha, \beta ; \Omega)$ is bounded and $\mathrm{L}^{2}(\Omega ; \mathcal{L}(\mathrm{Sym}, \mathrm{Sym}))$ is continuously imbedded in $\mathrm{H}^{-1}(\Omega ; \mathcal{L}(\mathrm{Sym}, \mathrm{Sym}))$, there exists a constant $c>0$ such that

$$
\left(\forall \mathbf{M} \in \mathfrak{M}_{2}(\alpha, \beta ; \Omega)\right) \quad\left\|u_{n}\right\|_{\mathrm{L}^{2}(\Omega)}+\left\|\mathbf{M} \nabla \nabla u_{n}\right\|_{\mathrm{H}^{-1}(\Omega ; \text { Sym })} \leq c\left\|f_{n}\right\|_{\mathrm{H}^{-2}(\Omega)} .
$$

Clearly, the same is true if we replace $\mathbf{M}$ and $\left(u_{n}\right)$ with tensor $\mathbf{O}$ and the corresponding sequence $\left(v_{n}\right)$, which implies that the series in definition of $d$ converges. In order to verify that $d$ is a metric, we shall only prove that $d(\mathbf{M}, \mathbf{O})=0$ implies $\mathbf{M}=\mathbf{O}$, as other properties are straightforward. The equality $d(\mathbf{M}, \mathbf{O})=0$ implies that for any $f \in \mathrm{H}^{-2}(\Omega)$, the solutions $u$ and $v$ of

$$
\left\{\begin{array} { l } 
{ \operatorname { d i v } \operatorname { d i v } ( \mathbf { M } \nabla \nabla u ) = f , } \\
{ u \in \mathrm { H } _ { 0 } ^ { 2 } ( \Omega ) }
\end{array} \quad \text { and } \quad \left\{\begin{array}{l}
\operatorname{div} \operatorname{div}(\mathbf{O} \nabla \nabla v)=f, \\
v \in \mathrm{H}_{0}^{2}(\Omega)
\end{array}\right.\right.
$$

satisfy $u=v$ and $\mathbf{M} \nabla \nabla u=\mathbf{O} \nabla \nabla v$ in $\Omega$. Indeed, by definition of $d$, this immediately follows for $f \in F$, and then for any $f \in \mathrm{H}^{-2}(\Omega)$ by density of set $F$ in $\mathrm{H}^{-2}(\Omega)$ and continuity of linear mappings $f \mapsto u$ and $f \mapsto v$ from $\mathrm{H}^{-2}(\Omega)$ to $\mathrm{H}_{0}^{2}(\Omega)$. For a set $\omega$ compactly embedded in $\Omega$ let us take $\varphi \in \mathrm{C}_{c}^{\infty}(\Omega)$ 
such that $\left.\varphi\right|_{\omega}=1$. If we take $f=\operatorname{div} \operatorname{div}\left(\mathbf{M} \nabla \nabla\left(\frac{1}{2} \mathbf{S x} \cdot \mathbf{x} \varphi(\mathbf{x})\right)\right)$, for $\mathbf{S} \in$ Sym arbitrary, this yields $\nabla \nabla u=\nabla \nabla v=\mathbf{S}$ in $\omega$, implying $\mathbf{M S}=\mathbf{O S}$ in $\omega$, and finally $\mathbf{M}=\mathbf{O}$, by arbitrariness of $\mathbf{S}$ and $\omega$.

It remains to prove that $\mathrm{H}$-convergence is equivalent to the convergence in this metric space. Assume that sequence $\left(\mathbf{M}^{m}\right)$ in $\mathfrak{M}_{2}(\alpha, \beta ; \Omega)$ H-converges to $\mathbf{M}$ in $\mathfrak{M}_{2}(\alpha, \beta ; \Omega)$, and let $\left(u_{n}^{m}\right),\left(u_{n}\right)$ be the sequences of solutions of

$$
\left\{\begin{array} { l } 
{ \operatorname { d i v } \operatorname { d i v } ( \mathbf { M } ^ { m } \nabla \nabla u _ { n } ^ { m } ) = f _ { n } , } \\
{ u _ { n } ^ { m } \in \mathrm { H } _ { 0 } ^ { 2 } ( \Omega ) }
\end{array} \quad \text { and } \quad \left\{\begin{array}{l}
\operatorname{div} \operatorname{div}\left(\mathbf{M} \nabla \nabla u_{n}\right)=f_{n}, \\
u_{n} \in \mathrm{H}_{0}^{2}(\Omega)
\end{array}\right.\right.
$$

respectively. Since $\left(\mathbf{M}^{m}\right)$ H-converges to $\mathbf{M}$ it follows

$$
u_{n}^{m} \longrightarrow u_{n} \text { in } \mathrm{H}_{0}^{2}(\Omega), \quad \mathbf{M}^{m} \nabla \nabla u_{n}^{m} \longrightarrow \mathbf{M} \nabla \nabla u_{n} \text { in } \mathrm{L}^{2}(\Omega ; \mathrm{Sym})
$$

and by Rellich compactness theorem we have strong convergences $u_{n}^{m} \rightarrow u_{n}$ in $\mathrm{L}^{2}(\Omega)$ and $\mathbf{M}^{m} \nabla \nabla u_{n}^{m} \rightarrow \mathbf{M} \nabla \nabla u_{n}$ in $\mathrm{H}^{-1}(\Omega ;$ Sym $)$, which implies $d\left(\mathbf{M}^{m}, \mathbf{M}\right) \rightarrow 0$.

In order to prove the converse statement, let a sequence $\left(\mathbf{M}^{m}\right)$ and $\mathbf{M}$ belong to $\mathfrak{M}_{2}(\alpha, \beta ; \Omega)$ and $d\left(\mathbf{M}^{m}, \mathbf{M}\right) \rightarrow 0$. We take an arbitrary $f \in \mathrm{H}^{-2}(\Omega)$ and a sequence $\left(f_{n^{\prime}}\right) \subseteq F$ strongly converging to $f$ in $\mathrm{H}^{-2}(\Omega)$. Let $u, u^{m}, u_{n^{\prime}}$ and $u_{n^{\prime}}^{m}$ be solutions of

$$
\begin{aligned}
& \left\{\begin{array} { l } 
{ \operatorname { d i v } \operatorname { d i v } ( \mathbf { M } \nabla \nabla u ) = f , } \\
{ u \in \mathrm { H } _ { 0 } ^ { 2 } ( \Omega ) , }
\end{array} \quad \left\{\begin{array}{l}
\operatorname{div} \operatorname{div}\left(\mathbf{M}^{m} \nabla \nabla u^{m}\right)=f, \\
u^{m} \in \mathrm{H}_{0}^{2}(\Omega),
\end{array}\right.\right. \\
& \left\{\begin{array} { l } 
{ \operatorname { d i v } \operatorname { d i v } ( \mathbf { M } \nabla \nabla u _ { n ^ { \prime } } ) = f _ { n ^ { \prime } } , } \\
{ u _ { n ^ { \prime } } \in \mathrm { H } _ { 0 } ^ { 2 } ( \Omega ) , }
\end{array} \quad \left\{\begin{array}{l}
\operatorname{div} \operatorname{div}\left(\mathbf{M}^{m} \nabla \nabla u_{n^{\prime}}^{m}\right)=f_{n^{\prime}}, \\
u_{n^{\prime}}^{m} \in \mathrm{H}_{0}^{2}(\Omega),
\end{array}\right.\right.
\end{aligned}
$$

respectively. For any $n^{\prime} \in \mathbf{N}$ the sequences $\left(u_{n^{\prime}}^{m}\right)_{m}$ and $\left(\mathbf{M}^{m} \nabla \nabla u_{n^{\prime}}^{m}\right)_{m}$ are bounded in $\mathrm{H}_{0}^{2}(\Omega)$ and $\mathrm{L}^{2}(\Omega$; Sym $)$, respectively, and therefore converge weakly on a subsequence. However, from $d\left(\mathbf{M}^{m}, \mathbf{M}\right) \rightarrow 0$ it follows that, for every $n^{\prime} \in \mathbf{N}, u_{n^{\prime}}^{m} \rightarrow u_{n^{\prime}}$ in $\mathrm{L}^{2}(\Omega)$ and $\mathbf{M}^{m} \nabla \nabla u_{n^{\prime}}^{m} \rightarrow \mathbf{M} \nabla \nabla u_{n^{\prime}}$ in $\mathrm{H}^{-1}(\Omega ; \mathrm{Sym})$, which implies the convergence of whole sequences:

$$
u_{n^{\prime}}^{m} \longrightarrow u_{n^{\prime}} \text { in } \mathrm{H}_{0}^{2}(\Omega), \quad \mathbf{M}^{m} \nabla \nabla u_{n^{\prime}}^{m} \longrightarrow \mathbf{M} \nabla \nabla u_{n^{\prime}} \text { in } \mathrm{L}^{2}(\Omega ; \mathrm{Sym}),
$$

as $m \rightarrow \infty$.

If we subtract the equations for $u$ and $u_{n^{\prime}}$, we get

$$
\left\{\begin{array}{l}
\operatorname{div} \operatorname{div}\left(\mathbf{M} \nabla \nabla\left(u-u_{n^{\prime}}\right)\right)=f-f_{n^{\prime}}, \\
u-u_{n^{\prime}} \in \mathrm{H}_{0}^{2}(\Omega)
\end{array}\right.
$$

and similarly for $u^{m}$ and $u_{n^{\prime}}^{m}$ :

$$
\left\{\begin{array}{l}
\operatorname{div} \operatorname{div}\left(\mathbf{M}^{m} \nabla \nabla\left(u^{m}-u_{n^{\prime}}^{m}\right)\right)=f-f_{n^{\prime}} \\
u^{m}-u_{n^{\prime}}^{m} \in \mathrm{H}_{0}^{2}(\Omega)
\end{array}\right.
$$

Since $\left(f_{n^{\prime}}\right)$ strongly converges to $f$, the well-posedness result for these problems ensure that $u_{n^{\prime}} \rightarrow u$ in $\mathrm{H}_{0}^{2}(\Omega)$ and thus $\mathbf{M} \nabla \nabla u_{n^{\prime}} \rightarrow \mathbf{M} \nabla \nabla u$ in $\mathrm{L}^{2}(\Omega ; \mathrm{Sym})$, as well as $u_{n^{\prime}}^{m} \rightarrow u^{m}$ in $\mathrm{H}_{0}^{2}(\Omega)$ and thus $\mathbf{M}^{m} \nabla \nabla u_{n^{\prime}}^{m} \rightarrow \mathbf{M}^{m} \nabla \nabla u^{m}$ in $\mathrm{L}^{2}(\Omega ; \mathrm{Sym})$, uniformly in $m$ as $n^{\prime} \rightarrow \infty$. Here, for the last convergence we have also used boundedness of the sequence $\left(\mathbf{M}^{m}\right)$ in $\mathrm{L}^{\infty}(\Omega ; \mathcal{L}(\mathrm{Sym}, \mathrm{Sym}))$. 
Together with (2.3) this implies

$$
\begin{aligned}
u^{m} & \longrightarrow u \text { in } \mathrm{H}_{0}^{2}(\Omega), \\
\mathbf{M}^{m} \nabla \nabla u^{m} & \longrightarrow \mathbf{M} \nabla \nabla u \quad \text { in } \quad \mathrm{L}^{2}(\Omega ; \mathrm{Sym}),
\end{aligned}
$$

i. e. $\mathbf{M}^{m}$ H-converges to $\mathbf{M}$, by arbitrariness of $f$. Indeed, for an arbitrary $f \in \mathrm{H}^{-2}(\Omega)$ and $\varepsilon>0$, the above (uniform) convergences imply that the first and the third term on the right-hand side of the inequality

$$
\begin{aligned}
& \left.\quad\right|_{\mathrm{H}^{-2}(\Omega)}\left\langle f, u^{m}-u\right\rangle_{\mathrm{H}_{0}^{2}(\Omega)}|\leq|_{\mathrm{H}^{-2}(\Omega)}\left\langle f, u^{m}-u_{n^{\prime}}^{m}\right\rangle_{\mathrm{H}_{0}^{2}(\Omega)} \mid \\
& \quad+\left.\right|_{\mathrm{H}^{-2}(\Omega)}\left\langle f, u_{n^{\prime}}^{m}-u_{n^{\prime}}\right\rangle_{\mathrm{H}_{0}^{2}(\Omega)}|+|_{\mathrm{H}^{-2}(\Omega)}\left\langle f, u_{n^{\prime}}-u\right\rangle_{\mathrm{H}_{0}^{2}(\Omega)} \mid
\end{aligned}
$$

can be made $\varepsilon$ small for $n^{\prime}$ large enough, i. e.

$$
\left|\mathrm{H}^{-2}(\Omega)\left\langle f, u^{m}-u\right\rangle_{\mathrm{H}_{0}^{2}(\Omega)}\right| \leq 2 \varepsilon+\left.\right|_{\mathrm{H}^{-2}(\Omega)}\left\langle f, u_{n^{\prime}}^{m}-u_{n^{\prime}}\right\rangle_{\mathrm{H}_{0}^{2}(\Omega)} \mid
$$

is valid for every $m$ and $n^{\prime}$ large enough. Taking the limit as $m \rightarrow \infty$, from (2.3) and arbitrariness of $\varepsilon$ and $f$, we get the first convergence in (2.4), while the second one can be derived similarly.

\section{Corrector results}

This section is devoted to corrector results. Their goal is to improve convergence of $\nabla \nabla u_{n}$ by adding correctors, and ending up with strong convergence, instead of the weak one given by the definition of H-convergence.

Definition 2. Let $\left(\mathbf{M}^{n}\right)$ be a sequence of tensors in $\mathfrak{M}_{2}(\alpha, \beta ; \Omega)$ that H-converges to a limit $\mathbf{M}^{*}$. For $1 \leq i, j \leq 2$ let $\left(w_{n}^{i j}\right)_{n}$ be a sequence of oscillating test functions satisfying

$$
w_{n}^{i j} \longrightarrow \frac{1}{2} x_{i} x_{j} \text { in } \mathrm{H}^{2}(\Omega), \quad \operatorname{div} \operatorname{div}\left(\mathbf{M}^{n} \nabla \nabla w_{n}^{i j}\right) \longrightarrow g_{i j} \text { in } \mathrm{H}_{\mathrm{loc}}^{-2}(\Omega),
$$

where $g_{i j}$ are some elements of $\mathrm{H}_{\mathrm{loc}}^{-2}(\Omega)$. The tensor $\mathbf{W}^{n}$ with components $\mathbf{W}_{i j k m}^{n}:=\left[\nabla \nabla w_{n}^{k m}\right]_{i j}$ is called the corrector.

It is important to note that functions $\left(w_{n}^{i j}\right)_{1 \leq i, j \leq N}$ are not uniquely defined. However, for any other family of such functions, it is easy to see that their difference converges strongly to zero in $\mathrm{H}^{2}(\Omega)$, and similar holds for the corrector tensors.

Lemma 2. Let $\left(\mathbf{M}^{n}\right)$ be a sequence of tensors in $\mathfrak{M}_{2}(\alpha, \beta ; \Omega)$ that H-converges to a tensor $\mathbf{M}^{*}$. A sequence of correctors $\left(\mathbf{W}^{n}\right)$ is unique in the sense that, for any two sequences of correctors $\left(\mathbf{W}^{n}\right)$ and $\left(\tilde{\mathbf{W}}^{n}\right)$, their difference $\left(\mathbf{W}^{n}-\tilde{\mathbf{W}}^{n}\right)$ converges strongly to zero in $\mathrm{L}_{\text {loc }}^{2}(\Omega ; \mathcal{L}(\mathrm{Sym}, \mathrm{Sym}))$.

Proof. For $1 \leq i, j \leq 2$, let $\left(w_{n}^{i j}\right)_{n}$ and $\left(\widetilde{w}_{n}^{i j}\right)_{n}$ be two sequence satisfying (3.1) and let $\varphi \in \mathrm{C}_{c}^{\infty}(\Omega)$. Using coercivity of $\mathbf{M}^{n}$, and integrating by parts two 
times we obtain:

$$
\begin{aligned}
\alpha \| \varphi( & \left.\nabla \nabla w_{n}^{i j}-\nabla \nabla \widetilde{w}_{n}^{i j}\right) \|_{L^{2}(\Omega ; \text { Sym })}^{2} \leq \int_{\Omega} \varphi^{2} \mathbf{M}^{n} \nabla \nabla\left(w_{n}^{i j}-\widetilde{w}_{n}^{i j}\right): \nabla \nabla\left(w_{n}^{i j}-\widetilde{w}_{n}^{i j}\right) d \mathbf{x} \\
= & { }_{\mathrm{H}_{\mathrm{loc}}^{-2}(\Omega)}\left\langle\operatorname{div} \operatorname{div}\left(\mathbf{M}^{n} \nabla \nabla\left(w_{n}^{i j}-\widetilde{w}_{n}^{i j}\right), \varphi^{2}\left(w_{n}^{i j}-\widetilde{w}_{n}^{i j}\right)\right\rangle_{H_{c}^{2}(\Omega)}\right. \\
& +{ }_{\mathrm{H}_{\mathrm{loc}}^{-1}(\Omega)}\left\langle\operatorname{div}\left(\mathbf{M}^{n} \nabla \nabla\left(w_{n}^{i j}-\widetilde{w}_{n}^{i j}\right)\right), \nabla\left(\varphi^{2}\right)\left(w_{n}^{i j}-\widetilde{w}_{n}^{i j}\right)\right\rangle_{H_{c}^{1}(\Omega)} \\
& \left.-{ }_{\mathrm{L}_{\mathrm{loc}}^{2}(\Omega)}\left\langle\mathbf{M}^{n} \nabla \nabla\left(w_{n}^{i j}-\widetilde{w}_{n}^{i j}\right), \nabla\left(w_{n}^{i j}-\widetilde{w}_{n}^{i j}\right) \nabla\left(\varphi^{2}\right)\right)\right\rangle_{L_{c}^{2}(\Omega) .}
\end{aligned}
$$

Each term on the right hand side tends to zero when $n \rightarrow \infty$, the first one because of the assumption (3.1), while the second one and the third one converge to zero by the Rellich compactness theorem. Thus, we deduce that $\nabla \nabla\left(w_{n}^{i j}-\widetilde{w}_{n}^{i j}\right)$ converges strongly to zero in $\mathrm{L}_{\text {loc }}^{2}(\Omega$; Sym $)$, which proves the statement.

Lemma 3. Let $\left(\mathbf{M}^{n}\right)$ be a sequence of tensors in $\mathfrak{M}_{2}(\alpha, \beta ; \Omega)$ that $H$-converges to a limit $\mathbf{M}^{*}$, and $\mathbf{W}^{n}$ the corresponding sequence of correctors. Then

$$
\begin{array}{r}
\mathbf{W}^{n} \longrightarrow \mathbf{I}_{4} \quad \text { in } \quad \mathrm{L}^{2}(\Omega ; \mathcal{L}(\text { Sym, Sym })), \\
\mathbf{M}^{n} \mathbf{W}^{n} \longrightarrow \mathbf{M}^{*} \quad \text { in } \quad \mathrm{L}^{2}(\Omega ; \mathcal{L}(\text { Sym, Sym })), \\
\left(\mathbf{W}^{n}\right)^{T} \mathbf{M}^{n} \mathbf{W}^{n} \longrightarrow \mathbf{M}^{*} \quad \text { in } \quad \mathrm{D}^{\prime}(\Omega ; \mathcal{L}(\text { Sym }, \text { Sym })) .
\end{array}
$$

Proof. The first convergence is a consequence of the definition of correctors. The second one follows from the definition of $\mathrm{H}$-convergence, and the third one from the compactness by compensation result applied to the components of $\left(\mathbf{W}^{n}\right)^{T}$ and $\mathbf{M}^{n} \mathbf{W}^{n}$.

In the next theorem we clarify in what sense correctors transform a weak convergence into the strong one.

Theorem 9. Let $\left(\mathbf{M}^{n}\right)$ be a sequence of tensors in $\mathfrak{M}_{2}(\alpha, \beta ; \Omega)$ which H-converges to $\mathbf{M}^{*}$. For $f \in \mathrm{H}^{-2}(\Omega)$, let $\left(u_{n}\right)$ be the sequence of solutions to

$$
\left\{\begin{array}{l}
\operatorname{div} \operatorname{div}\left(\mathbf{M}^{n} \nabla \nabla u_{n}\right)=f \\
u_{n} \in \mathrm{H}_{0}^{2}(\Omega)
\end{array}\right.
$$

Let $u$ be the weak limit of $\left(u_{n}\right)$ in $\mathrm{H}_{0}^{2}(\Omega)$, i.e., the solution of the homogenized equation

$$
\left\{\begin{array}{l}
\operatorname{div} \operatorname{div}\left(\mathbf{M}^{*} \nabla \nabla u\right)=f \\
u \in \mathrm{H}_{0}^{2}(\Omega)
\end{array}\right.
$$

Then, if we denote $r_{n}:=\nabla \nabla u_{n}-\mathbf{W}^{n} \nabla \nabla u$, where $\mathbf{W}^{n}$ is the corrector, it holds that $\left(r_{n}\right)$ converges strongly to zero in $\mathrm{L}_{\text {loc }}^{1}(\Omega ; \mathrm{Sym})$. 
Proof. Let $\varphi \in C_{c}^{\infty}(\Omega)$, and let $\left(v_{m}\right)$ be a sequence in $C_{c}^{\infty}(\Omega)$ such that $v_{m} \rightarrow u$ in $\mathrm{H}_{0}^{2}(\Omega)$. Since $\mathbf{M}^{n}$ is coercive we have

$$
\begin{aligned}
& \alpha\left\|\varphi\left(\nabla \nabla u_{n}-\mathbf{W}^{n} \nabla \nabla v_{m}\right)\right\|_{\mathrm{L}^{2}(\Omega ; \text { Sym })}^{2} \\
& \leq \int_{\Omega} \varphi^{2} \mathbf{M}^{n}\left(\nabla \nabla u_{n}-\mathbf{W}^{n} \nabla \nabla v_{m}\right):\left(\nabla \nabla u_{n}-\mathbf{W}^{n} \nabla \nabla v_{m}\right) d \mathbf{x} \\
& =\int_{\Omega} \varphi^{2} \mathbf{M}^{n} \nabla \nabla u_{n}: \nabla \nabla u_{n} d \mathbf{x}-\int_{\Omega} \varphi^{2} \mathbf{M}^{n} \nabla \nabla u_{n}: \mathbf{W}^{n} \nabla \nabla v_{m} d \mathbf{x}- \\
& \quad-\int_{\Omega} \varphi^{2} \mathbf{M}^{n} \mathbf{W}^{n} \nabla \nabla v_{m}: \nabla \nabla u_{n} d \mathbf{x}+\int_{\Omega} \varphi^{2}\left(\mathbf{W}^{n}\right)^{T} \mathbf{M}^{n} \mathbf{W}^{n} \nabla \nabla v_{m}: \nabla \nabla v_{m} d \mathbf{x} .
\end{aligned}
$$

As $n \rightarrow+\infty$, the first term on the right hand side converges by Theorem 4 , while the second and the third term converge by the compensated compactness result. The last term converges by Lemma 3, leading to

$$
\begin{aligned}
\limsup _{n \rightarrow \infty}\left\|\varphi\left(\nabla \nabla u_{n}-\mathbf{W}^{n} \nabla \nabla v_{m}\right)\right\|_{\mathrm{L}^{2}(\Omega ; \mathrm{Sym})}^{2} & \\
\leq & \frac{1}{\alpha} \int_{\Omega} \varphi^{2} \mathbf{M}^{*} \nabla \nabla\left(u-v_{m}\right): \nabla \nabla\left(u-v_{m}\right) d \mathbf{x} .
\end{aligned}
$$

If $u$ is smooth (in that case we can choose $v_{m}=u$ ), the proof is finished. If $u$ is not smooth, than after taking limit as $n \rightarrow+\infty$ in the estimate ( $c$ is generic constant below)

$$
\begin{aligned}
& \| \varphi\left(\nabla \nabla u_{n}-\mathbf{W}^{n} \nabla \nabla u\right) \|_{\mathrm{L}^{1}(\Omega ; \mathrm{Sym})} \\
& \leq\left\|\varphi\left(\nabla \nabla u_{n}-\mathbf{W}^{n} \nabla \nabla v_{m}\right)\right\|_{\mathrm{L}^{1}(\Omega ; \mathrm{Sym})}+\left\|\varphi \mathbf{W}^{n}\left(\nabla \nabla v_{m}-\nabla \nabla u\right)\right\|_{\mathrm{L}^{1}(\Omega ; \mathrm{Sym})} \\
& \leq c \varphi \varphi\left(\nabla \nabla u_{n}-\mathbf{W}^{n} \nabla \nabla v_{m}\right) \|_{\mathrm{L}^{2}(\Omega ; \mathrm{Sym})} \\
& \quad+\left\|\varphi \mathbf{W}^{n}\right\|_{\mathrm{L}^{2}(\Omega ; \mathcal{L}(\mathrm{Sym}, \mathrm{Sym}))}\left\|\nabla \nabla v_{m}-\nabla \nabla u\right\|_{\mathrm{L}^{2}(\Omega ; \mathrm{Sym})},
\end{aligned}
$$

we get

$$
\begin{aligned}
& \limsup _{n \rightarrow \infty} \| \varphi\left(\nabla \nabla u_{n}-\mathbf{W}^{n} \nabla \nabla u\right) \|_{\mathrm{L}^{1}(\Omega ; \mathrm{Sym})} \\
& \quad \leq c\left(\left\|\varphi\left(\nabla \nabla u-\nabla \nabla v_{m}\right)\right\|_{\mathrm{L}^{2}(\Omega ; \mathrm{Sym})}+\left\|\nabla \nabla v_{m}-\nabla \nabla u\right\|_{\mathrm{L}^{2}(\Omega ; \mathrm{Sym})}\right),
\end{aligned}
$$

and finally

$$
\begin{aligned}
& \limsup _{n \rightarrow \infty}\left\|\varphi\left(\nabla \nabla u_{n}-\mathbf{W}_{n} \nabla \nabla u\right)\right\|_{\mathrm{L}^{1}(\Omega ; \mathrm{Sym})} \\
& \quad \leq c \limsup _{m \rightarrow \infty}\left\|\varphi\left(\nabla \nabla u-\nabla \nabla v_{m}\right)\right\|_{\mathrm{L}^{2}(\Omega ; \mathrm{Sym})}+c\left\|\nabla \nabla u-\nabla \nabla v_{m}\right\|_{\mathrm{L}^{2}(\Omega ; \mathrm{Sym})}=0,
\end{aligned}
$$

which finishes the proof, by arbitrariness of $\varphi$.

\section{Conclusions}

We have proved a number of properties for $\mathrm{H}$-convergence in the context of the stationary plate equation and have given simpler proofs for some of them, which could otherwise be derived from homogenization results for general elliptic systems [15]. Clearly, one could push forward presented work by considering 
some other important questions such as small-amplitude homogenization and G-closure problem for stationary plate equation, problem of boundary homogenization in more general terms [6], or considering homogenization theory for vibrating plates (see [4]). We leave this open for some future work. Let us remark that this shall also pave the way for possible applications of the homogenization theory in optimal design problems for stationary and vibrating plates [12].

\section{References}

[1] G. Allaire. Shape optimization by the homogenization method. Springer, 2001.

[2] N. Antonić and N. Balenović. Optimal design for plates and relaxation. Mathematical Communications, 4:111-119, 1999.

[3] N. Antonić and N. Balenović. Homogenisation and optimal design for plates. Zeitschrift fur Angewandte Mathematik und Mechanik, 80:757-758, 2000.

[4] N. Antonić, N. Balenović and M. Vrdoljak. Optimal design for vibrating plates. Zeitschrift fur Angewandte Mathematik und Mechanik, 80:783-784, 2000.

[5] N. Antonić, A. Raguž and M. Vrdoljak. Homogenisation of nonlinear elliptic systems. In M. Rogina et al.(Ed.), Applied mathematics and computation, pp. 81-90, Department of Mathematics, University of Zagreb, 2001.

[6] D. Blanchard, A. Gaudiello and T. Mel'nyk. Boundary homogenization and reduction of dimension in a Kirchhoff-love plate. SIAM Journal Mathematical Analysis, 39(6):1764-1787, 2008. https://doi.org/10.1137/070685919.

[7] M. Bukal and I. Velčić. On the simultaneous homogenization and dimension reduction in elasticity and locality of $\Gamma$-closure. Calculus of Variations and Partial Differential Equations, 56(3):59, 2017. https://doi.org/10.1007/s00526017-1167-z.

[8] A. Damlamian and M. Vogelius. Homogenization limits of the equations of elasticity in thin domains. SIAM Journal on Mathematical Analysis, 18(2):435451, 1987. https://doi.org/10.1137/0518034.

[9] L.V. Gibiansky and A.V. Cherkaev. Design of composite plates of extremal rigidity. In A. Cherkaev and R. Kohn(Eds.), Topics in the Mathematical Modelling of Composite Materials, pp. 95-137, Boston, MA, 1997. Birkhäuser, Boston. https://doi.org/10.1007/978-1-4612-2032-9_5. The article is the translation of an article originally written in Russian and published as the report of Ioffe PhysicoTechnical Institute, Academy of Sciences of USSR, publication 914, Leningrad, 1984

[10] K.A. Lurie, A.V. Cherkaev and A.V. Fedorov. Regularization of optimal design problems for bars and plates I-II. Journal of Optimization Theory and Applications, 37(4):499-543, 1982. https://doi.org/10.1007/BF00934953.

[11] F. Murat and L. Tartar. H-Convergence. In Topics in the Mathematical Modelling of Composite Materials, volume 31 of Progress in Nonlinear Differential Equations and Their Applications, 1997. https://doi.org/10.1007/9781-4612-2032-9_3. The article is the translation of notes originally written in French. Mimeographed notes. Séminaire d'Analyse Fonctionnelle et Numérique de l'Université d'Alger, 1977/78 
[12] R. Prakash. Optimal control problem for the time-dependent Kirchoff-Love plate in a domain with rough boundary. Asymptotic Analysis, 81(3-4):337-355, 2013. https://doi.org/10.3233/ASY-2012-1132.

[13] L. Tartar. An introduction to the homogenization method in optimal design. In A. Cellina and A. Ornelas(Eds.), Optimal shape design, volume 1740 of Lecture Notes in Mathematics, pp. 47-156. Springer, Berlin, 2000. https://doi.org/10.1007/BFb0106742.

[14] L. Tartar. The general theory of homogenization. Springer, 2009.

[15] V.V. Zhikov, S.M. Kozlov, O.A. Oleinik and Kha T'en Ngoan. Averaging and Gconvergence of differential operators. Russian Mathematical Surveys, 34(5):69147, 1979. https://doi.org/10.1070/RM1979v034n05ABEH003898. 